EPJ Web of Conferences 71, 00010 (2014)

DOI: 10.1051/epjconf/20147100010

(C) Owned by the authors, published by EDP Sciences, 2014

\title{
Supersymmetry searches at CMS
}

\section{Summary of selected Supersymmetry searches of CMS at $\sqrt{s}=8 \mathrm{TeV}$}

\author{
Dr. Pedrame Bargassa ${ }^{1, a}$ \\ ${ }^{1}$ LIP - Av. Elias Garcia 14-1, 1000-149 Lisboa, Portugal
}

\begin{abstract}
The accumulation of $19 \mathrm{fb}^{-1}$ of data at $8 \mathrm{TeV}$ has been a unique window for $\mathrm{Su}$ persymmetry searches at the LHC, allowing the CMS collaboration to search for specific supersymmetric particles. This article covers the search for supersymmetric particles in a variety of production- and decay-modes as well as channels. It focuses on the search for the lightest scalar top (stop) in the single lepton channel, stop which might be the only observable in the case where Supersymmetry is realized in nature, and which can play a unique role in "naturalness".
\end{abstract}

\section{Introduction}

Supersymmetric (Susy) theories [1] predict the existence of a scalar partner for each standard model fermion. The search for a rather low energy Susy has special interest in view of the discovery of a light Higgs boson [2], which constrains the Susy parameter space: While a Susy Higgs is being seriously constrained in the case of a $\tau$-phobic Higgs and for a light supersymmetric tau (stau), more possibilities subsist for the realization of Susy in the case of a light stop as an example, see figure 1. In the following sections, different searches of Susy performed by the CMS collaboration at $\sqrt{s}=8$ $\mathrm{TeV}$ and in different final states are presented; in each case, different interpretations are provided in terms of sensitivity to the mass of searched supersymmetric particles. These searches consider strong production processes and cascade decays producing many jets and missing transverse momentum $\left(B_{T}\right)$ from unobserved, weakly interacting particles. For all searches, the Lightest Supersymmetric Particle (LSP) is assumed to be the lightest neutralino $\tilde{\chi}_{1}^{0}$, itself assumed to be stable. For the searches in final states with 0,1 , and 3 leptons, the searches are general in scope and motivated by models of new physics, including Susy. An exclusive and generic search for stop pair production is presented at the end of this article.

\section{Search in final states with 0 lepton}

One of the main searches for Susy in hadronic final states performed by CMS [4] is mainly characterized by the requirement of at least three jets with transverse momentum $p_{T}>50 \mathrm{GeV} / \mathrm{c}$, high $H_{T}$ defined as $\left|\Sigma_{\text {jets }} p_{T}(j e t)\right|$, high $H_{T}^{\text {miss }}$ defined as $\left|-\Sigma_{\text {jets }} p_{T}(\vec{j} e t)\right|$, and high $H_{T}$. To increase the sensitivity of the search for generic final states containing jets, $H_{T}$ and $H_{T}^{\text {miss }}$, a simultaneous examination

ae-mail: bargassa@cern.ch 


\section{EPJ Web of Conferences}
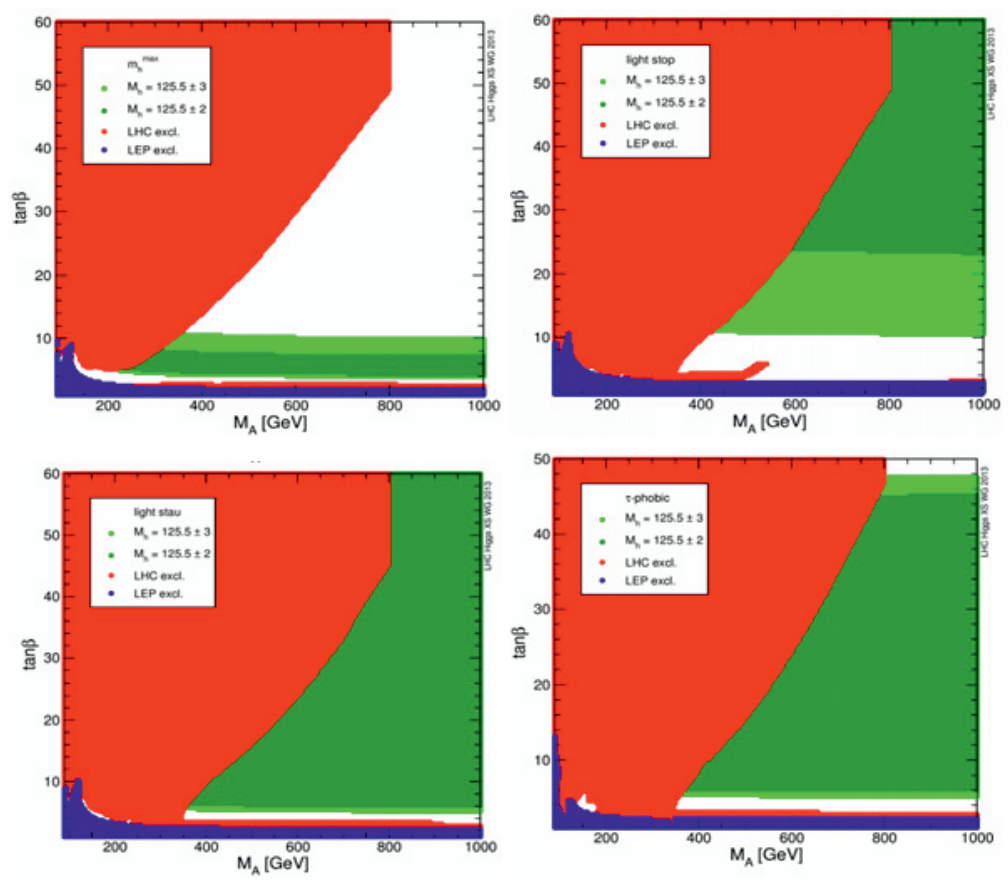

Figure 1. Excluded regions in the Susy parameter space $\left(\tan \beta, \mathrm{M}_{A}\right)$ by different experiments and searches, and for various Susy scenarios [3].

of the data is done of 36 exclusive bins of jet multiplicity, $H_{T}$ and $H_{T}^{\text {miss }}$, the prediction of the background from the Standard Model (SM) being driven by data. No excess of data is observed versus the predicted SM background (see figure 2); this absence is interpreted as 95\% Confidence Limit [5] (CL) exclusion of signal models, as function of the mass of searched supersymmetric particles. The production- and decay-modes of Susy particles for which there is most sensitivity in this final state and for this type of selection are direct production of squark $(\tilde{q})$ and gluino $(\tilde{g})$ followed by their decay into a final state with the LSP:

- $p p \rightarrow \tilde{q} \tilde{q}$ with $\tilde{q} \rightarrow q \tilde{\chi_{1}^{0}}$,

- $p p \rightarrow \tilde{g} \tilde{g}$ with $\tilde{g} \rightarrow q \tilde{q} \tilde{\chi}_{1}^{0}$.

Since the mass of involved particles are unknown, a scan of the sensitivity of this search is performed as function of $\left(\mathrm{m}(\tilde{q}), \mathrm{m}\left(\tilde{\chi_{1}^{0}}\right)\right)$ and $\left(\mathrm{m}(\tilde{g}), \mathrm{m}\left(\tilde{\chi_{1}^{0}}\right)\right)$ for the two cases mentioned above, and presented in figure 2: Light-flavor squarks are excluded with a mass below $\sim 800 \mathrm{GeV} / \mathrm{c}^{2}$, this for the LSP mass below $\sim 300 \mathrm{GeV} / \mathrm{c}^{2}$; the gluino is excluded with a mass below $\sim 1.2 \mathrm{TeV} / \mathrm{c}^{2}$, this for the LSP mass below $\sim 500 \mathrm{GeV} / \mathrm{c}^{2}$.

\section{Search in final states with 1 lepton}

The general CMS search for Susy in final states with one lepton (electron and muon) [6] requires an isolated lepton with $p_{T}>20 \mathrm{GeV} / \mathrm{c}$, at least three jets with $p_{T}>40 \mathrm{GeV} / \mathrm{c}$, high $H_{T}$ and $H_{T}$, and at least two jets tagged as stemming from a $b$ quark; the presence of a second lepton is vetoed. The main 

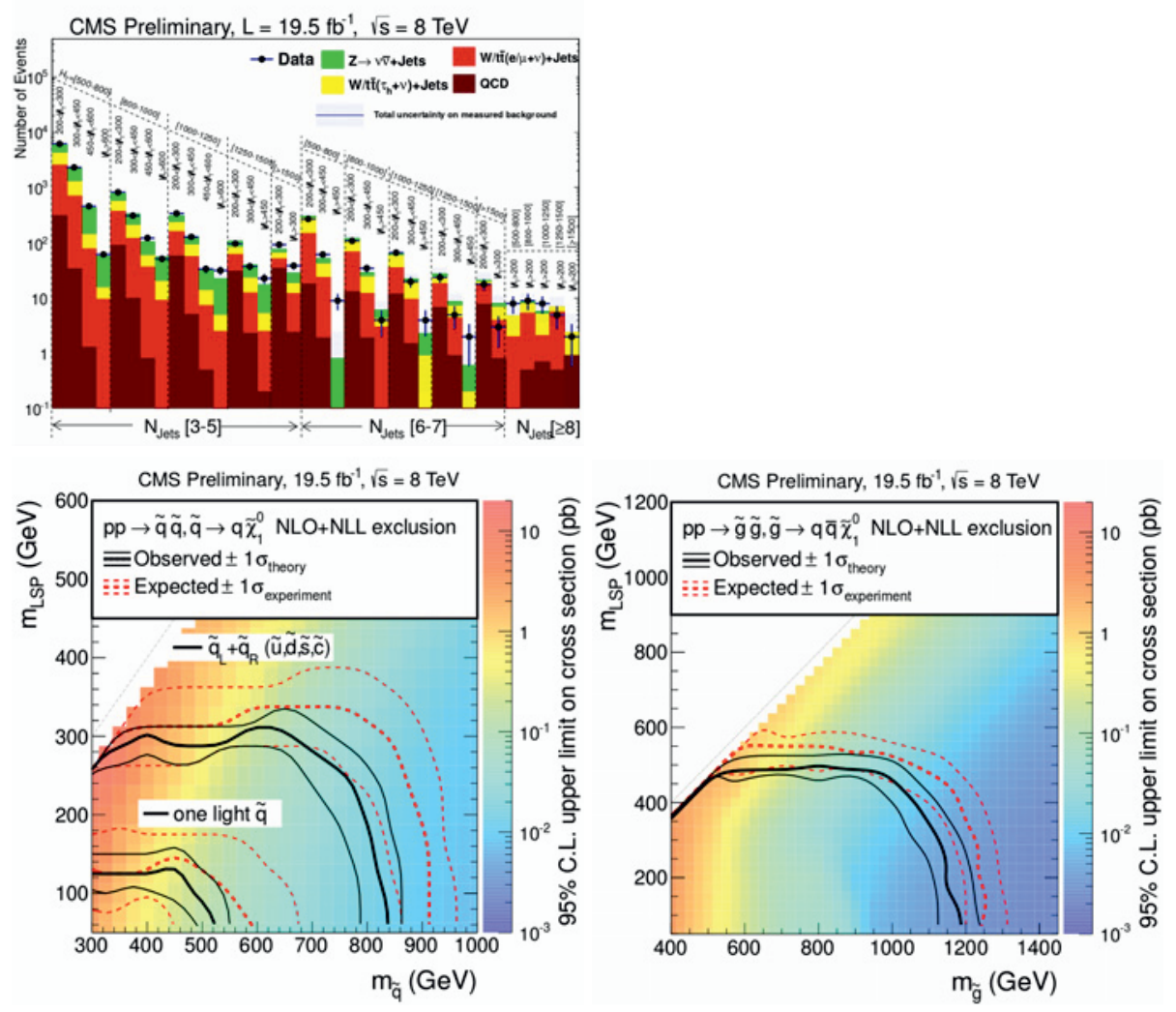

Figure 2. Results of the Susy search in the hadronic finale state [4]: Top: SM background expectation and data events in the $36\left(N_{\text {Jets }}, H_{T}, H_{T}^{\text {miss }}\right)$ bins. Bottom: Sensitivity of the search in the $\left(\mathrm{m}(\tilde{q}), \mathrm{m}\left(\chi_{1}^{0}\right)\right)$ (left) and $\left(\mathrm{m}(\tilde{g}), \mathrm{m}\left(\tilde{\chi_{1}^{0}}\right)\right)($ right $)$ planes.

background is the production of a $\mathrm{W}$ boson associated with jets stemming from Initial State Radiation (ISR). The similarity between the $p_{T}$ of a neutrino and of a charged lepton in the decays of the W boson is used to predict the $B_{T}$ distribution and infer a background prediction in data; this prediction is validated in a background dominated region of data, defined by requiring only the presence of at least 4 jets, and extended to the signal region defined by requiring at least 6 jets and 2 b-tagged jets; the observed and predicted events are compared in different $H_{T}$ and $H_{T}$ bins in both regions. As can be observed in figure 3, the data is well described by the prediction of the SM background, the absence of excess being interpreted in terms of $95 \% \mathrm{CL}$ exclusion limit. One of the signal models which is the most sensitive to this final state is the direct gluino production $p p \rightarrow \tilde{g} \tilde{g}$ followed by $\tilde{g} \rightarrow \overline{t \bar{t}} \chi_{1}^{0}$ where the branching fraction of four $\mathrm{W}$ bosons decaying in a final state with a single lepton is $\sim 44 \%$. Under this hypothesis, and because of the presence of the lepton whose threshold is lower than jets, the sensitivity to the direct gluino production is extended at low $\Delta m=m(\tilde{g})-m\left(\tilde{\chi_{1}^{0}}\right)$ region, but also for the low LSP mass where the sensitivity to the gluino is extended to $\geq 1.3 \mathrm{TeV} / \mathrm{c}^{2}$. 

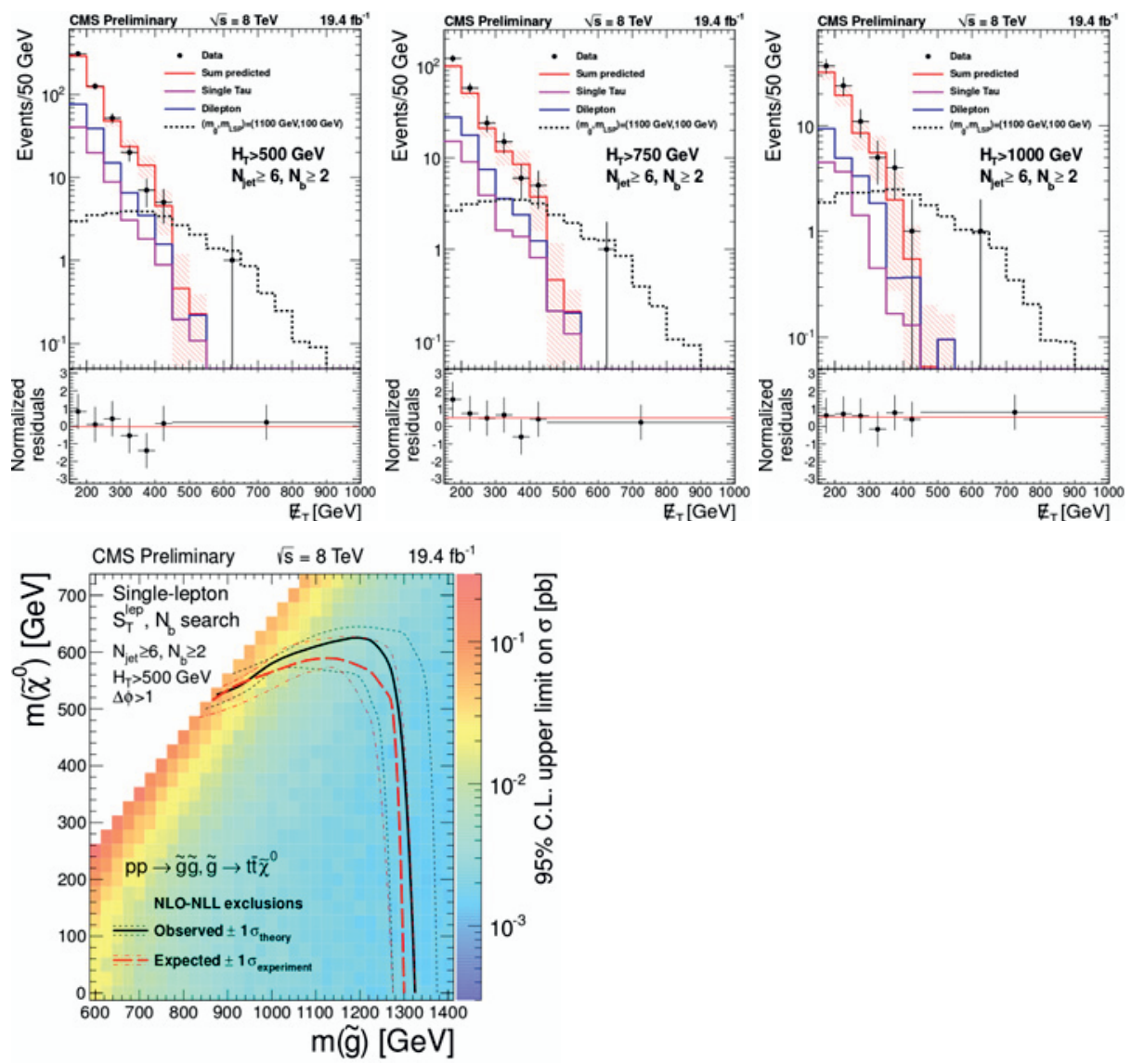

Figure 3. Results of the Susy search in the hadronic finale state [6]: Top: Data and background prediction in the signal region, in different bins of $H_{T}$. Bottom: Sensitivity of the search in the $\left(\mathrm{m}(\tilde{g}), \mathrm{m}\left(\tilde{\chi_{1}^{0}}\right)\right)$ plane.

\section{Search in final states with 3 leptons}

The general CMS search for Susy in this final state [7] requires three leptons with $p_{T}>20,10,10$ $\mathrm{GeV} / \mathrm{c}$. Due to the low level of the multijet background, the selection cuts on $H_{T}$ and $H_{T}$ are relatively low, mainly aimed at reducing the contribution of $\mathrm{Z}$ bosons associated with jets where these latter stem from low $p_{T}$ ISR and where the low $B_{T}$ is due to instrumental and reconstruction effects. At least two jets are required, one of which should be b-tagged. To further reduce the contribution of the $\mathrm{Z}$ boson production, the selection on the number of jets and b-tagged jets, $H_{T}$ and $H_{T}$ is tightened when the invariant mass of two same flavor and opposite sign leptons is reconstructed in the vicinity of the $\mathrm{Z}$ mass. The result of the search of a signal beyond the SM in this final state can be interpreted in a variety of production- and decay-modes of supersymmetric objects, ranging from direct gluino to lightest supersymmetric bottom (sbottom $\tilde{b_{1}}$ ) production, with decays involving stops, or sbottoms, or the lightest chargino $\tilde{\chi_{1}^{ \pm}}$, or the second lightest neutralino $\tilde{\chi_{2}^{0}}$; each scenario is reported in figure 4 with the corresponding sensitivity of the search as function of the mass of the involved particles. 


\section{ICNFP 2013}
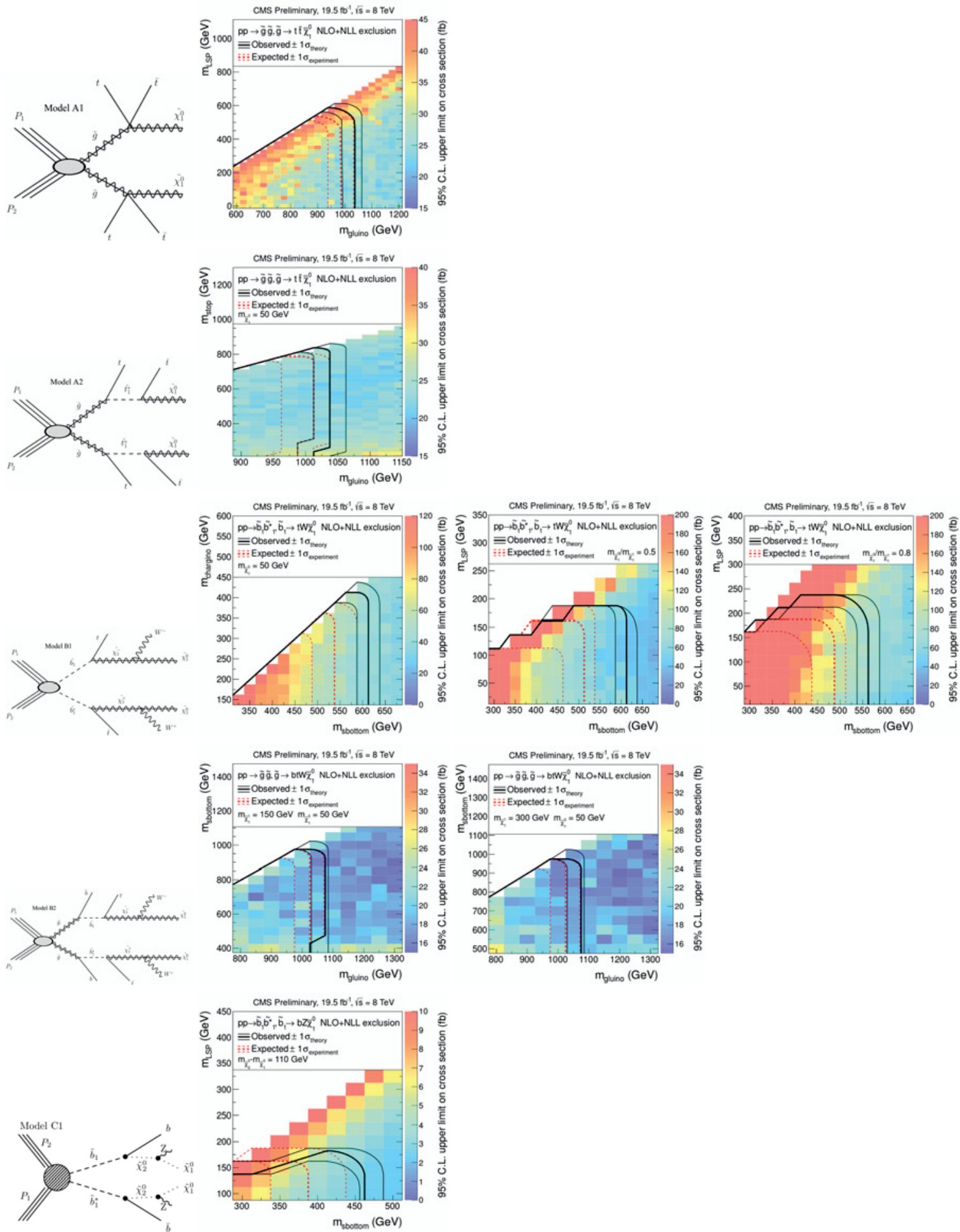

Figure 4. Results of the Susy search in the hadronic finale state [7]. Each row refers to the considered productionand decay-mode of Susy particles (left column), followed by its sensitivity as function of the mass of the involved particles. 


\section{Search for direct stop production in final states with 1 lepton}

The searches covered in previous sections are motivated by models of new physics, which are possible to interpret in many models, including Susy. Being frequently based on selections requiring high $H_{T}$ and $B_{T}$, they are most of the time sensitive to the production of rather high mass Susy objects such as gluino and light-flavor squarks, sometimes involving long decay chains. This section covers the exclusive search for direct stop production in final states with one lepton $(e / \mu)$, purposefully searching for a supersymmetric object which is probably at the bottom of the Susy mass chain.

Because of the large mass of the standard model top quark, the mixing between its chiral supersymmetric partners is the largest among all squarks; therefore the lightest supersymmetric partner of the top quark, $\tilde{t_{1}}$ (stop), might be the lightest squark producible at the LHC. Furthermore, the stop sector $\tilde{t_{1,2}}$ is the Susy sector most sensitive to the Higgs mass [8]. Finally, the measure of the Cold Dark Matter (CDM) density [9] gives preference to a particle whose mass is close to the one of the $\mathrm{CDM}$; in the framework of Susy, this can be the lightest stop with a mass close to the one of $\tilde{\chi_{1}^{0}}$, natural supersymmetric candidate for the CDM.

The CMS exclusive search for the direct production of the lightest stop in the single lepton final state [10] considers the decays of the lightest stop in $\tilde{b \chi_{1}^{ \pm}}$and $t^{(*)} \tilde{\chi_{1}^{0}}$, both decaying in $b W^{ \pm} \tilde{\chi_{1}^{0}}$. This search requires an electron or muon with $p_{T}>30 \mathrm{GeV} / \mathrm{c}$, at least four jets with at least one of them b-tagged, a $B_{T}$ larger than $100 \mathrm{GeV}$, and a transverse invariant mass of the (lepton, $B_{T}$ ) system larger than $150 \mathrm{GeV}$. Then discriminating kinematic and topological variables are fed into Boosted Decision Tree (BDT) in order to maximize the separation between signal and background. The variation of the kinematics of a large variety of $\left(\tilde{t}_{1}, \tilde{\chi}_{1}^{0}\right)$ signal points is taken into account by training different BDTs for signal with different $\Delta m=m\left(\tilde{t_{1}}\right)-m\left(\tilde{\chi_{1}^{0}}\right)$. In figure 5 we report the output of the BDT for both decay modes, and for each decay mode for signals with different $\Delta m$; for each decay mode, we can observe that signal points with a high $\Delta m$ is better separated from the SM background. The observed absence of data versus the SM background for the different BDT trainings is interpreted as 95\% CL exclusion of signal as a function of the mass of the lightest stop and LSP, this for the two decay modes (see figure 5). In the $b \tilde{\chi}_{1}^{ \pm}$decay mode, three different hypothesis are made about the mass of the lightest chargino (figure 5), while in the $t^{(*)} \tilde{\chi}_{1}^{0}$ decay mode, the case for unpolarized and right-handed tops are considered in the interpretation of the results. Across the different hypothesis on the decay mode, and within them, different kinematic configurations, stop masses below $\sim 650$ $\mathrm{GeV} / \mathrm{c}^{2}$ are excluded for LSP masses below $\sim 230 \mathrm{GeV} / \mathrm{c}^{2}$. It has to be noted that in the case of the $t^{(*)} \chi_{1}^{0}$ decay mode, there is a loss of sensitivity along the $m\left(\tilde{t_{1}}\right)-m\left(\tilde{\chi_{1}^{0}}\right)=m(t)$ line below which the top is virtual.

\section{Conclusions}

During the year 2012, the CMS detector has collected $19 \mathrm{fb}^{-1}$ of data at a center of mass energy of $8 \mathrm{TeV}$. An extensive search program has been pursued through many final states, with sensitivity to many Susy particles. General searches exclude the gluino with a mass $\lesssim 1.2 \mathrm{TeV} / \mathrm{c}^{2}$ for LSP mass $\lesssim 700 \mathrm{GeV} / \mathrm{c}^{2}$; they exclude light-flavor squarks with a mass $\lesssim 850 \mathrm{GeV} / \mathrm{c}^{2}$ for LSP mass $\leq 300$ $\mathrm{GeV} / \mathrm{c}^{2}$. These same searches exclude the lightest sbottom in a direct production mode for masses below $600 \mathrm{GeV} / \mathrm{c}^{2}$ for LSP masses below 200 to $600 \mathrm{GeV} / \mathrm{c}^{2}$, depending on the mass hypothesis on the lightest nautralino and chargino. The amount of collected data allowed an exclusive search for the direct production of the lightest stop, here presented in the single lepton final state; stop masses below $650 \mathrm{GeV} / \mathrm{c}^{2}$ are excluded for LSP mass below $250 \mathrm{GeV} / \mathrm{c}^{2}$, this across two different decay modes of the stop and different mass hypothesis about the particles involved. 

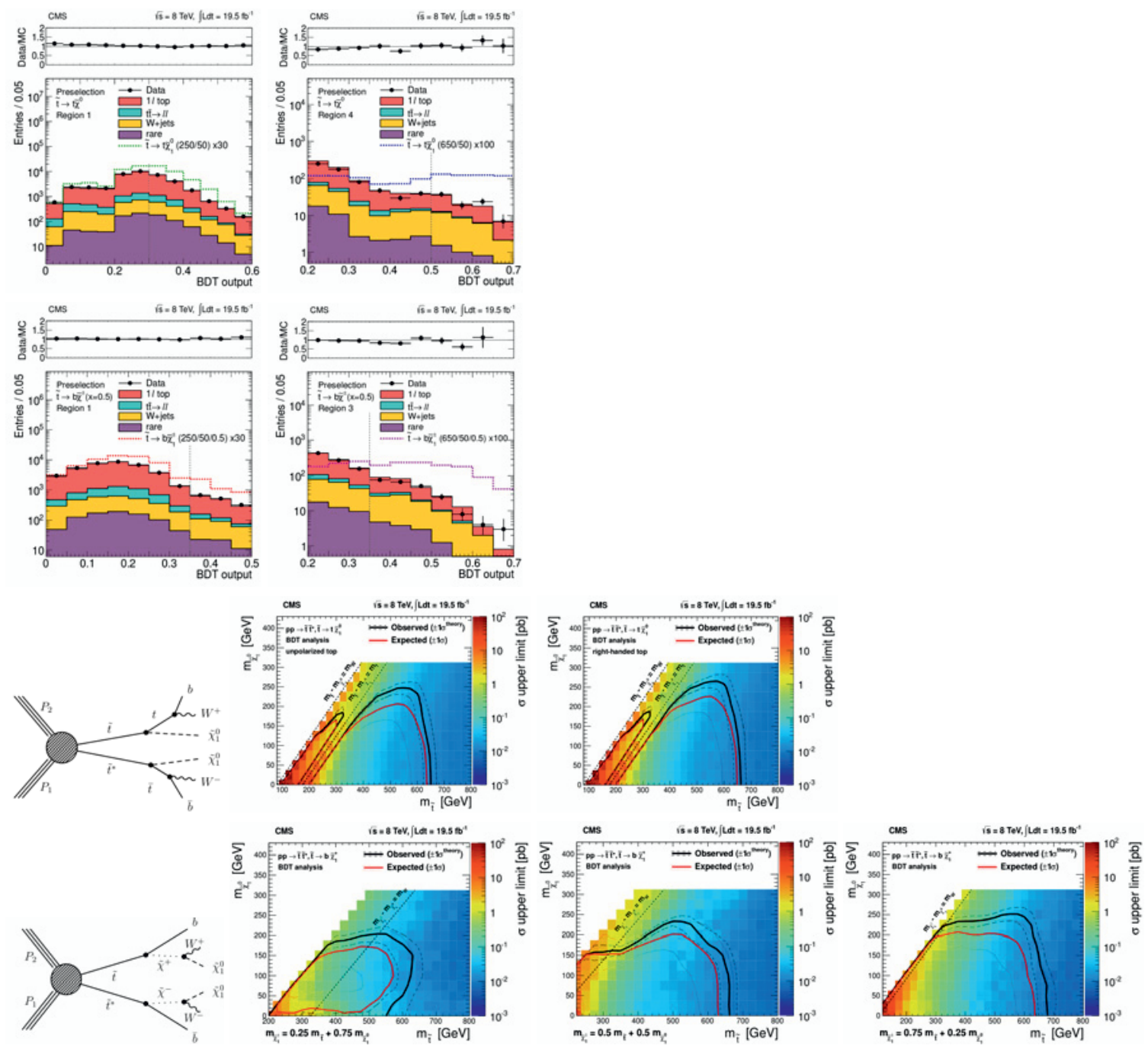

Figure 5. Results of the stop search in the single lepton final state [10]. First row: BDT output distribution for data, SM background and signal points with $\Delta m=200$ and $400 \mathrm{GeV} / \mathrm{c}^{2}$ in the $\tau_{\chi_{1}^{0}}$ decay mode. Second row: Same for the $b \tilde{\chi_{1}^{ \pm}}$decay mode. Third row: $t^{(*)} \tilde{\chi}_{1}^{0}$ decay mode: Sensitivity of the single lepton stop search as function of the stop and LSP masses for an unpolarized (center) and right-handed (right) top. Fourth row: $b \tilde{\chi}_{1}^{ \pm}$ decay mode: Sensitivity of the single lepton stop search as function of the stop and LSP masses for $m\left(\tilde{\chi_{1}^{ \pm}}\right)=$ $0.25 m\left(\tilde{t_{1}}\right)+0.75 m\left(\tilde{\chi_{1}^{0}}\right), 0.5 m\left(\tilde{t_{1}}\right)+0.5 m\left(\tilde{\chi_{1}^{0}}\right)$ and $0.75 m\left(\tilde{t_{1}}\right)+0.25 m\left(\tilde{\chi_{1}^{0}}\right)$.

\section{References}

[1] S.P. Martin, Perspectives on Supersymmetry, World Scientific, Singapore, 1998; revised version [arXiv:hep-ph/9709356v4].

[2] Chatrchyan et al., PLB 716 30-61 (2012). Aad et al., PLB 716 1-29 (2012). 
EPJ Web of Conferences

[3] The LHC Higgs Cross Section Working Group, Handbook of LHC Higgs Cross. Sections: 3. Higgs Properties, arXiv:1307.1347

[4] CMS collaboration, CMS-PAS-SUS-13-012

[5] T. Junk, Nucl. Instrum. Methods A 434, 435 (1999)

[6] CMS collaboration, CMS-PAS-SUS-13-007

[7] CMS collaboration, CMS-PAS-SUS-13-008

[8] Demina et al., PRD 62, 35011

[9] Boehm et al., PRD 62, 35012

[10] CMS collaboration, CMS-PAS-SUS-13-011 